\title{
Erecteur a dépression (Vacuum erection device) : Mise au point
}

\author{
D. DelaVierRe
}

Service d'Urologie- Andrologie, CHR d'ORLEANS

\section{RESUME}

L'érecteur à dépression (Vacuum erection device) est une méthode thérapeutique palliative de l'insuffisance érectile utilisant le vide et un anneau constricteur. Il provoque une érection dont les caractéristiques sont différentes de l'érection naturelle en raison d'une réduction du flux sanguin pénien et de la température cutanée, d'une congestion des tissus extra-caverneux, d'une bascule de la verge et d'un blocage de l'éjaculation. Les résultats de la littérature nous permettent d'estimer qu'environ 30 à $60 \%$ des patients à qui une période d'essai a été proposée achètent l'érecteur. 60 à $80 \%$ des acheteurs continuent d'utiliser le système à moyen terme. Les abandons semblent moins fréquents qu'avec les auto-injections intra-caverneuses. Les inconvénients du système, outre ceux liés aux caractéristiques de l'érection, sont dominés par les douleurs et la survenue de pétéchies ou ecchymoses au niveau de la verge. Actuellement, au même titre que les autoinjections intra-caverneuses, l'érecteur concerne les patients ne réagissant pas aux nouveaux traitements oraux ou ne pouvant prendre ceux-ci en raison d'une contre-indication.

Mots-clés: Dysfonction érectile, impuissance sexuelle, érecteur à dépression.
Nous dédions ce travail au Docteur Perry W. NADIG, disparu en 1997, qui fut un pionnier dans l'utilisation de l'érecteur à dépression et contribua à sa reconnaissance par le monde scientifique.

\section{INTRODUCTION}

L'érecteur à dépression (Vacuum erection device) est une méthode médicale de traitement de l'insuffisance érectile utilisant le vide et un anneau constricteur. En raison de son aspect mécanique ce système a été mal accepté par le milieu médical. En 1996 les recommandations de l'American Urological Association, reconnaissant l'érecteur comme une option thérapeutique (au même titre que les injections intra-caverneuses et les prothèses péniennes) ont donné à cette méthode une reconnaissance scientifique indiscutable [17]. Actuellement, au même titre que les auto-injections intracaverneuses, il concerne les patients ne réagissant pas aux nouveaux traitements oraux ou ne pouvant prendre ceux-ci en raison d'une contre-indication.

\section{HISTORIQUE}

Le premier brevet d'invention d'un érecteur à dépression a été déposé aux USA en 1917 par Otto Lederer [12]. Cette technique a ensuite été négligée par le corps médical qui ne l'a pas prise au sérieux et n'a réalisé aucun travail scientifique sur le sujet. 
A partir de 1961 David Osbon, devenu partiellement impuissant vers 60 ans, a développé un nouveau système qu'il utilisera lui-même pendant plus de 20 ans. Ce système est devenu en 1983 l'Erecaid System [27].

En 1985 Roy Witherington a présenté au congrès de l'American Urological Association une communication orale consacrée à cet érecteur [26] mais c'est en 1986 que Perry Nadig a publié dans Urology le premier article sur cette méthode non invasive capable de provoquer et de maintenir une érection [20].

\section{PRINCIPES DU FONCTIONNEMENT}

L'utilisation d'un érecteur à dépression répond à deux principes :

1. Une érection survient si le pénis est placé dans un système en dépression.

2. Cette érection se maintient si un anneau constricteur est placé autour de la base du pénis.

\section{Base expérimentale}

En 1989 Diederichs étudie les effets de la dépression sur le pénis de 9 singes sous anesthésie [6]. Un cylindre est placé autour du pénis et la ponction d'un corps caverneux permet de mesurer la pression intra-caverneuse. A l'état flaccide cette pression est de 7 à $15 \mathrm{~cm}$ $\mathrm{H}_{2} \mathrm{O}$.

Quand une dépression de -100 à $-200 \mathrm{~cm} \mathrm{H}_{2} \mathrm{O}$ est créée dans le cylindre, la pression intra caverneuse tombe entre -60 et $-160 \mathrm{~cm} \mathrm{H}_{2} \mathrm{O}$ puis elle se rétablit en 30 à 60 secondes. Durant ce rétablissement, le pénis augmente de volume et le sang afflue dans les corps caverneux. Quand la pression atmosphérique est rétablie dans le cylindre, la pression intracaverneuse augmente de 5 à $10 \mathrm{~cm} \mathrm{H}_{2} \mathrm{O}$ pendant 3 à 6 secondes tandis que la détumescence s'installe.

Si un anneau constricteur est placé à la base du pénis avant la dépression, les effets de celleci sont identiques. Mais quand la pression atmosphérique est rétablie dans le cylindre, la pression intra-caverneuse monte entre 60 et $120 \mathrm{~cm} \mathrm{H} \mathrm{H}_{2} \mathrm{O}$. Cette pression est stable jusqu'à l'ablation de l'anneau puis chute en 2 à 6 secondes quand cette ablation intervient.

La surface de section pénienne (mesurée par échographie) augmente au cours de l'érection provoquée de $150 \%$ par rapport à la flaccidité. La simple mise en place d'un anneau constricteur à la base de la verge flaccide (sans dépression) n'entraîne aucune modification de la pression intra-caverneuse et de la surface de section pénienne.

Après injection intra-caverneuse de Papavérine la pression intra-caverneuse et la surface de section pénienne sont identiques à celles obtenues après dépression, mais la surface de section des corps caverneux est plus importante après Papavérine. En effet lors de la dépression la contraction persistante des fibres musculaires lisses limite l'élongation de l'albuginée des corps caverneux, mais par contre, les tissus extra-caverneux sont tumescents.

En conclusion de son étude, Diederichs précise que la tumescence induite par la dépression est passive et intervient sans relaxation des fibres musculaires lisses et sans libération de neurotransmetteurs.

\section{Principe d'utilisation chez l'homme}

L'érecteur à dépression comporte un cylindre en plastique transparent dont une extrémité est ouverte, une pompe fixée ou reliée par un raccord à l'autre extrémité et des anneaux constricteurs élastiques.

Un ou plusieurs anneaux sont glissés autour de l'extrémité ouverte du cylindre. Celle-ci et toute la verge sont enduites d'un lubrifiant hydrosoluble. Le cylindre est placé autour de la verge flaccide et appliqué contre le pubis et la région péri-pénienne afin de créer une chambre étanche. La pompe permet alors d'établir une dépression dans le cylindre. Nadig précise que la dépression nécessaire pour obtenir une érection rigide est au maximum de $200 \mathrm{~mm} \mathrm{Hg}$ [20]. Dans sa série le temps nécessaire pour obtenir cette érection, une fois la dépression créée, est de 3 à $7 \mathrm{mn}$. Dans celles de Witherington [27] et Sidi [21] ce temps est respectivement de $2.5 \mathrm{mn}$ en moyenne et $30 \mathrm{~s}$ à $2 \mathrm{mn}$. Quand l'érection est obtenue le ou les anneaux sont transférés autour de la verge. La pression atmosphérique est alors 
rétablie dans le cylindre grâce à une valve située sur la pompe puis le cylindre et retiré. L'érection se maintient et permet un rapport sexuel. Il n'est pas recommandé de garder l'anneau en place plus de $30 \mathrm{mn}$.

\section{Caractéristiques de cette érection pro- voquée}

L'érecteur à dépression entraîne une érection différente de l'érection naturelle en raison notamment de la constriction pénienne.

\section{a) Le flux sanguin pénien diminue}

La réduction du flux sanguin pénien a été mis en évidence par les travaux de Marmar [14], Katz [11] et Broderick [2]. Ceux-ci ont respectivement utilisés la pléthysmographie, l'injection intra-caverneuse de xénon radioactif et l'échodoppler des artères caverneuses. La réduction du flux sanguin pénien crée des conditions d'ischémie mais l'intensité et la durée de cette réduction pouvant entraîner des lésions ischémiques ne sont pas connues.

\section{b) La température cutanée de la verge diminue}

Ce refroidissement est la conséquence de la baisse du flux sanguin. Nadig le chiffre à $0.96^{\circ} \mathrm{C}$ en moyenne [20] et Marmar entre 0.5 et $1.1^{\circ} \mathrm{C}[14]$.

\section{c) Congestion des tissus extra-caverneux}

Les veines superficielles sont distendues, la verge est cyanosée. Nadig note une augmentation de la circonférence de la verge à l'angle péno-scrotal de $4.3 \mathrm{~cm}$ au lieu de $2.8 \mathrm{~cm}$ au cours d'une érection normale [20].

\section{d) Bascule de la verge}

Le pénis n'est rigide qu'en aval de l'anneau tandis que la partie proximale des corps caverneux reste flaccide.

\section{e) Blocage de l'éjaculation}

L'éjaculation peut être emprisonnée dans l'uretère en amont de l'anneau.

\section{RESULTATS}

\section{Interpretations et limites}

Avec l'érecteur à dépression, l'interprétation des résultats de la littérature est souvent difficile. Les critères de sélection des patients sont rarement précisés et il s'agit parfois de patients qui ne sont pas satisfaits des autoinjections intra-caverneuses. Dans l'analyse des résultats un certain nombre de patients sont souvent éliminés (patients perdus de vue, n'ayant pas répondu à un questionnaire, n'ayant pas acheté l'érecteur malgré la prescription). Enfin l'expression des résultats n'est pas standardisée. Ainsi différents critères sont utilisés : capacité d'obtention d'une érection suffisante pour une pénétration vaginale, satisfaction, nombre d'achats ou d'utilisateurs après une période de traitement à domicile.

A notre avis, les critères certes les plus sévères, mais également les plus fiables pour apprécier l'érecteur à dépression sont le nombre d'acheteurs ou d'utilisateurs après une période de traitement à domicile. Ce nombre est inférieur au nombre de patients capables d'obtenir une érection utilisable ou satisfaits et la différence peut même être importante. Ainsi Gilbert a noté que $83 \%$ de ses patients étaient capables d'obtenir une érection utilisable mais seulement $27 \%$ étaient satisfaits et $16 \%$ ont acheté l'érecteur [8].

\section{Resultats globaux}

Dans notre analyse de la littérature nous avons éliminé les séries comportant moins de 40 patients et avons retenu 11 séries de 1990 à 1997 (Tableaux 1 et 2).

7 séries (Tableau 1) analysent les résultats en fonction du pourcentage de patients utilisant le système après un certain suivi. Si on excepte Earle ce pourcentage est compris entre 57 et $80 \%$ après un suivi de 3 à 29 mois. La série de Earle (seulement $19 \%$ d'utilisateurs) concernait des patients qui n'étaient pas satisfaits des auto-injections intra-caverneuses [7].

4 séries (Tableau 2) analysent les résultats en fonction du nombre d'achats après une période 
Tableau 1 : EFFICACITE (en fonction du nombre d'utilisateurs)

\begin{tabular}{lcccc}
\hline & $\begin{array}{c}\text { Nombre de } \\
\text { patients }\end{array}$ & $\begin{array}{c}\text { Nombre de } \\
\text { patients évalués }\end{array}$ & Suivi & \% d'utilisateurs \\
\hline Sidi 1990 [21] & 127 & 100 & 7,9 mois & $79 \%$ \\
Turner 1992 [24] & 45 & 45 & 12 mois & $80 \%$ \\
Cokson 1993 [4] & 238 & 161 & 3 mois & $69 \%$ \\
Baltaci 1995 [1] & & 115 & 29 mois & $70 \%$ \\
Earle 1996 [7] & 60 & 6149 & 12,8 mois & $59 \%$ \\
Lewis 1997 [13] & 34777 & 43 & $?$ & $19 \%$ \\
Giuliano 1997 [9] & 175 & 5847 & 27 mois & $76,8 \%$ \\
& & 77 & & $57 \%$ \\
\hline
\end{tabular}

Tableau 2 : EFFICACITE (en fonction du nombre d'achats après une période d'essai)

Nombre de patients évalués Nombre d'achats

(période d'essai)

$\begin{array}{lcc}\text { Gould 1992 [10] } & 46 & 65 \%(3 \text { mois }) \\ \text { Gilbert 1992 [8] } & 45 & 16 \%(3 \text { mois }) \\ \text { Meinhardt 1992 [16] } & 72 & 31 \% \text { (3 semaines) } \\ \text { Vrijhof 1994 [25] } & 47 & 28 \% \text { (2 semaines) }\end{array}$

d'essai initiale. 16 à $65 \%$ des patients ont acheté l'érecteur après une période d'essai de 2 semaines à 2 mois. Les séries de Gilbert [8] et Meinhardt [16] concernaient des patients traités auparavant par d'autres méthodes (notamment les auto-injections intra-caverneuses) sans succès.

$\mathrm{Au}$ vu de ces résultats on peut estimer qu'environ 30 à $60 \%$ de patients achètent l'érecteur après une période d'essai initiale et 60 à $80 \%$ des acheteurs continuent d'utiliser le système à moyen terme.

\section{Résultats en fonction des indications}

Peu de travaux étudient les résultats en fonction de chaque étiologie.

Après ablation de prothèses péniennes, notamment en raison d'infections, l'érecteur peut donner de bons résultats $[18,19]$.

Denil signale que presque 50\% d'une série de traumatisés médullaires et de leur épouse restaient satisfaits du système après 6 mois d'utilisation [5].

\section{Abandons (Tableau 3)}

20 à $40 \%$ des patients abandonnent le système à court ou moyen terme. Les raisons le plus souvent invoquées par les patients pour refuser ou abandonner l'érecteur sont non seulement la guérison ou l'inefficacité du système, mais également les effets secondaires, des problèmes techniques, le refus de la partenaire, des problèmes conjugaux, le rejet du système en raison de son aspect instrumental encombrant et du manque de naturel, enfin son coût excessif $[1,4,8,9,13,16,21,25]$.

\section{ECHECS, INCIDENTS ET COMPLICATIONS}

\section{Echecs}

Ils sont liés à l'impossibilité d'obtenir ou de maintenir une rigidité suffisante.

Une rigidité incomplète peut être due à plusieurs causes : impossibilité de créer une 


\begin{tabular}{|c|c|c|c|c|}
\hline $\begin{array}{l}\text { Nbre de } \\
\text { Patients }\end{array}$ & Abandons & Guérison & Inefficacité & $\begin{array}{c}\text { Refus de la } \\
\text { partenaire }\end{array}$ \\
\hline
\end{tabular}

$\begin{array}{lcccccc}\text { Giuliano [9] } & 77 & 33(43 \%) & 15,3 \% & 38,6 \% & 15,4 \% & 21,5 \% \\ \text { Lewis [13] } & 5847 & 1357(23 \%) & 10,8 \% & 40,9 \% & 20,6 \% & ?\end{array}$

chambre étanche autour de la verge (une lubrification abondante voire le rasage des poils pubiens y remédient), dépression insuffisante ou trop brève (une attente de quelques minutes est nécessaire), pénis trop gros en regard du cylindre.

Une rigidité fugace est due à une constriction insuffisante et nécessite d'utiliser un anneau plus serré.

Avant d'obtenir une érection satisfaisante et d'acheter l'appareil une période d'essai est nécessaire [27]. Cette période est précédée d'une information complète et suivie d'une évaluation précise [8].

\section{Incidents et complications}

a) Douleurs (pendant la dépression et après obtention de l'érection) $[4,8,9,21$, $24,27]$

Elles sont fréquentes (10 à $30 \%$ des patients) et d'intensité variable (parfois simple sensation d'inconfort). Pour les prévenir il est indispensable de créer lentement la dépression.

b) Pétéchies, contusions cutanées et ecchymoses [4, 9, 21, 27]

Elles sont également fréquentes (jusqu'à $30 \%$ des patients).

c) Blocage de l'éjaculation [1, 4, 21, 24]

Il survient chez 20 à $40 \%$ des patients avec parfois des douleurs par distension de l'urètre bulbaire [27].

d) Engourdissement et refroidissement de la verge sont fréquents.

\section{COMPARAISON ENTRE L'ERECTEUR ET LES AUTO-INJECTIONS}

Les patients de Soderdahl [22], qui ont successivement utilisé les 2 méthodes, préfèrent en majorité les auto-injections, notamment ceux âgés de moins de 60 ans, souffrant de dysfonction érectile depuis moins de 1 an ou prostatectomisés. Toutefois $58 \%$ des patients de Gould ayant utilisé les auto-injections avec succès, ont opté pour l'érecteur après un essai de 3 mois [10].

Turner à propos de 2 séries comparatives note que la fréquence d'utilisation et le taux de succès des 2 méthodes sont comparables mais le taux d'abandons est moins important avec l'érecteur [24].

Les 2 méthodes peuvent se compléter, l'érecteur provoquant facilement une érection sur une verge précédemment tumescente grâce à une injection intra-caverneuse [3, 15, 22].

\section{CONCLUSION}

En raison de son efficacité et de son innocuité, l'érecteur à dépression appartient à l'arsenal thérapeutique palliatif de la dysfonction érectile. Au même titre que les auto-injections intra-caverneuses il concerne les patients ne réagissant pas aux nouveaux traitements oraux ou ne pouvant prendre ceux-ci en raison d'une contre-indication. Il est également utile dans l'étude des incurvations de la verge notamment dans la maladie de La Peyronie $[23,28]$. 


\section{REFERENCES}

1. BALTACI S., AYDOS K., KOSAR A., ANAFARTA K.: Treating erectile dysfunction with a vacuum tumescence device : a retrospective analysis of acceptance and satisfaction. Br.J.Urol., 1995, 76 : 757-760

2. BRODERICK G.A., MCGAHAN J.P., STONE A.R., DEVERE WHITE R. : The hemodynamics of vacuum constriction erections : assessment by color doppler ultrasound. J.Urol., 1992, $147: 57-61$

3. CHEN J., GODSCHALK M.F., KATZ P.G., MULLIGAN T. : Combining intracavernous injection and external vacuum as teatment for erectile dysfunction. J.Urol., 1995, 153: 1476-1477

4. COKSON M.S., NADIG P.W. : Long-term results with vacuum constriction device. J.Urol., 1993, 149 : 290294

5. DENIL J., OHL D.A., SMYTHE C. : Vacuum erection device in spinal cord injured men : patient and partner satisfaction. Arch.Phys.Med.Rehabil., 1996, 77 : 750-753

6. DIEDERICHS W., KAULA N.F, LUE T.F. ET TANAGHO E.A. : The effect of subatmospheric pressure on the simian penis. J. Urol., 1989142 : 10871089

7. EARLE C.M., SEAH M., COULDEN S.E., STUCKEY B.G., KEOGH E.J. : The use of the vacuum erection device in the management of erectile impotence. Int.J.Impot.Res., 1996, 8 : 237-240

8. GILBERT H.W., GINGELL J.C. : Vacuum constriction devices : second-line conservative treatment for impotence. Br.J.Urol.,1993, $70: 81-83$

9. GIULIANO F., AMAR E., THIOUNN N. et al. : Etude rétrospective de l'efficacité et de l'acceptabilité du vacuum pour le traitement des troubles de l'érection. Prog.Urol., 1997, 7 Sup.1 : 10A

10. GOULD J.E., SWITTERS D.M., BRODERICK G.A., DEVERE WHITE R.W. : External vacuum devices : a clinical comparison with pharmacologic erections. World J.Urol. 1992, $10: 68-70$

11. KATZ P.G., HADEN H.T., MULLIGAN T. ET ZASLER N.D. : The effect of vacuum devices on penible hemodynamics. J. Urology , 1990, 143 : 55-56

12. LEDERER 0. : Surgical device, U.S. Patent number 1, 1917, 225, 341 : Mar 8,

13. LEWIS R.W., WITHERINGTON R. : External vacuum therapy for erectile dysfunction : use and results. World J.Urol., 1997, 15 : 78-82

14. MARMAR J.L., DEBENEDICTS T.J. ET PRAISS D.E. : Penile plethysmography on impotent men using vacuum constrictor devices. Urology. , 1988, 32 : 198-203.
15. MARMAR J.L., DEBENEDICTIS T.J. ET PRAISS D.E. : The use of vacuum constrictor device to augment a partiel erection following an intracavernous injection. J. Urology, 1988, $140:$ 975-979.

16. MEINHARDT W., LYCKLAMA A NIJEHOLT A.A.B., KROPMAN R.F., ZWARTENDIJK J. : The negative pressure device for erectile disorders : when does it fail ? J.Urol., 1993, 149 : 1285-1287

17. MONTAGUE D.K., BARADA J.H., BELKER A.M. et al. : Clinical guidelines panel on erectile dysfunction : summary report on the treatment of organic erectile dysfunction. Urol., 1996156 : 2007-2011

18. MOUL J.W. ET MACLEOD D.G. : Negative pressure devices in the explanted penile prosthesis population. J. Urology, 1989, $142:$ 729-731

19. NADIG P.W. : Utility of the vacuum-constriction device for men who have failed penile prostheses. J. Urology, 1986, $135: 232 \mathrm{~A}$

20. NADIG P.W., WARE J.C. ET BLUMOFF R. : Noninvasive device to produce and maintain an erectionlike state. Urology, 1986, $27: 126-131$

21. SIDI A.A., BECHER E.F., ZHANG G., LEWIS J.H. : Patient acceptance of and satisfaction with an external negative pressure device for impotence. J.Urol., 1990,144 : 1154-1156

22. SODERDAHL D.W., THRASHER J.B., HANSBERRY K.L. : Intracavernosal drug-induced erection therapy versus external vacuum devices in the treatment of erectile dysfunction. Br.J.Urol., 1997, 79 : 952-957

23. STRUB M.D. ET MICHAELS E.K. : Diagnostic artificial erection without corpus cavernosum injection. J. Urology, 1990, $143: 562$

24. TURNER L.A., ALTHOF S.E., LEVINE S.B., BODNER D.R., KURSH E.D., RESNICK M.I. : Twelve-month comparison of two treatments for erectile dysfunction : self-injection versus external vacuum devices. Urology, 1992, 39 : 139-144

25. VRIJHOF H.J.E.J., DELAERE K.P.J. : Vacuum constriction devices in erectile dysfunction : acceptance and effectiveness in patients with impotence of organic or mixed aetiology. Br.J.Urol., 1994, 74 : 102105

26. WITHERINGTON R. : The Osbon Erecaid System in the management of erectile impotence. Urology, $1985,133: 190 \mathrm{~A}$

27. WITHERINGTON R. : Vacuum constriction device for management of erectile impotence. J. Urology, $1989,141: 320-322$

28. YACHIA D. : Negative pressure induced erection for the assessment of impotent patients with Peyronie's disease. Br. J. Urology, 1990, 66 : 106-107 
Vacuum erection device

D. DELAVIERRE

Service d'Urologie-Andrologie, CHR d'ORLEANS

The vacuum erection device (VED) is a nonsurgical alternative for the treatment of erectile dysfunction.

This method is based on 2 phenomena :

1) an erection-like state occurs when the penis is placed in a vacuum

2) this state can be maintained by a constricting band placed around the base of the penis

The erection-like state thus obtained differs from a normal erection in the following ways :

1) blood flow into the penis decreases

2) penile skin temperature falls

3) congestion of extra-corporeal penile tissues occurs

4) the penis pivots at its base

5) the ejaculate is trapped in the proximal urethra

About 30 to $60 \%$ of men who trie the device at home purchase it for continued use. The others are unable to achieve sufficient rigidity, experience side-effects or dislike the use of the device despite good result. About 60 to 80 $\%$ of the men who purchased the device continue to use it regulary at short or middle term. The VED seems to have lesser drop-out rate than the self injection therapy.

The most frequently encountered side-effects of the device are pain, petechiaes, bruising and ecchymosis.

Key-words : Impotence, erectile dysfunction, vacuum erection device 